\title{
Lattice QCD calculation of the electroweak box diagrams for the kaon semileptonic decays
}

\author{
Peng-Xiang Ma $\odot,{ }^{1}$ Xu Feng $\odot,{ }^{1,2,3, *}$ Mikhail Gorchtein, ${ }^{4,5,6, \dagger}$ Lu-Chang Jin, ${ }^{7,8, \sharp}$ and Chien-Yeah Seng $\odot^{9, \S}$ \\ ${ }^{1}$ School of Physics and State Key Laboratory of Nuclear Physics and Technology, \\ Peking University, Beijing 100871, China \\ ${ }^{2}$ Collaborative Innovation Center of Quantum Matter, Beijing 100871, China \\ ${ }^{3}$ Center for High Energy Physics, Peking University, Beijing 100871, China \\ ${ }^{4}$ Helmholtz Institute Mainz, Mainz, 55128, Germany \\ ${ }^{5}$ GSI Helmholtzzentrum für Schwerionenforschung, Darmstadt 64291, Germany \\ ${ }^{6}$ Johannes Gutenberg University, Mainz. 55128, Germany \\ ${ }^{7}$ Department of Physics, University of Connecticut, Storrs, Connecticut 06269, USA \\ ${ }^{8}$ RIKEN-BNL Research Center, Brookhaven National Laboratory, Building 510, Upton, New York 11973 \\ ${ }^{9}$ Helmholtz-Institut für Strahlen- und Kernphysik and Bethe Center for Theoretical Physics, \\ Universität Bonn, 53115 Bonn, Germany
}

(Received 25 February 2021; accepted 11 May 2021; published 9 June 2021)

\begin{abstract}
We present a lattice QCD calculation of the axial $\gamma W$-box diagrams relevant for the kaon semileptonic decays. We utilize a recently proposed method, which connects the electroweak radiative corrections in Sirlin's representation to that in chiral perturbation theory. It allows us to use the axial $\gamma W$-box correction in the SU(3) limit to obtain the low energy constants for chiral perturbation theory. From first principles our results confirm the previously used low energy constants provided by the minimal resonance model with a significant reduction in uncertainties.
\end{abstract}

DOI: 10.1103/PhysRevD.103.114503

\section{INTRODUCTION}

In the Standard Model, the Cabibbo-KobayashiMaskawa (CKM) matrix is a three-generation quark mixing matrix which describes how the strength of the flavorchanging weak interaction in the leptonic sector is distributed among the three quark generations. The precise determination of the CKM matrix elements is of vital importance in the stringent test of CKM unitarity and search of new physics beyond the Standard Model. As quoted in the 2020 review by the Particle Data Group (PDG) [1], there exists a $\sim 3$ sigma deviation from unitarity in the first row of CKM matrix elements

$$
\left|V_{u d}\right|^{2}+\left|V_{u s}\right|^{2}+\left|V_{u b}\right|^{2}=0.9984(3)_{V_{u d}}(4)_{V_{u s}} .
$$

Here $\left|V_{u b}\right|^{2} \approx 1.5 \times 10^{-5}$ is negligibly small and thus only $\left|V_{u d}\right|$ and $\left|V_{u s}\right|$ play a role in the unitarity test.

\footnotetext{
xu.feng@pku.edu.cn

†gorshtey@uni-mainz.de

ljin.luchang@gmail.com

§cseng@hiskp.uni-bonn.de
}

Published by the American Physical Society under the terms of the Creative Commons Attribution 4.0 International license. Further distribution of this work must maintain attribution to the author(s) and the published article's title, journal citation, and DOI. Funded by SCOAP ${ }^{3}$.
The most precise determination of $\left|V_{u d}\right|=$ $0.97370(10)_{\exp +\text { nucl }}(10)_{\mathrm{RC}}$ quoted in the $2020 \mathrm{PDG}$ review [1] stems from the superallowed nuclear beta decays [2,3], with the first uncertainty arising from the experimental measurements and nuclear physics corrections and the second one from the electroweak radiative corrections (RCs). ${ }^{1}$ It is the update of the RCs from a dispersive analysis $[4,6]$ which makes the value of $\left|V_{u d}\right|$ about $2 \sigma$ smaller than that in the 2018 PDG review [7]. Very recently, the RCs to the $\pi_{\ell 3}$ decays were calculated using lattice QCD with the focus on the so-called axial $\gamma W$-box diagrams [8]. It allowed for a significant reduction of the hadronic uncertainty in the RCs, and provided an independent cross-check of the dispersion relation analysis of the neutron RCs [9]. In the future a direct lattice QCD calculation of the RCs to the neutron decay could help to further improve the determination of $\left|V_{u d}\right|$ [10].

The $\left|V_{u s}\right|$ can be determined from kaon, hyperon or tau decays, with kaon decays providing the best precision. Leptonic decays $K \rightarrow \mu \nu$ (denoted by $K_{\mu 2}$ ) combined with $\pi \rightarrow \mu \nu$ give access to the ratio $\left|V_{u s} / V_{u d}\right|$, whereas semileptonic decays $K \rightarrow \pi \ell \nu$ (denoted by $K_{\ell 3}$ ) give a handle

\footnotetext{
${ }^{1}$ Notice, however, that this quoted value does not include the contributions from several new nuclear corrections investigated in Refs. [4,5].
} 
on $\left|V_{u s}\right|$ independently. The traditional way of determining $\left|V_{u s}\right|$ relies on the experimental measurements of $K_{L}^{0} \rightarrow$ $\pi e \nu$ to avoid the isospin-breaking effects $\left(\pi^{0}-\eta\right.$ mixing $)$ in the charged kaon decays and the complication from the second (scalar) form factor present in the muonic decays. Nowadays, due to the high-statistics data collected in the experiments, the comparison between different decay modes is justified [11]. The decays including $K_{L}^{0} \rightarrow \pi \ell \nu$, $K^{ \pm} \rightarrow \pi^{0} \ell^{ \pm} \nu$ and $K_{S}^{0} \rightarrow \pi e \nu$ with $\ell=e, \mu$ are used to determine $\left|V_{u s}\right|$ via the master formula [1]

$\Gamma_{K \ell 3}=\frac{G_{F}^{2} m_{K}^{5}}{192 \pi^{3}} S_{\mathrm{EW}}\left(1+\delta_{K}^{\ell}+\delta_{S U 2}\right) C^{2}\left|V_{u s}\right|^{2} f_{+}^{2}(0) I_{K}^{\ell}$.

Here, $\Gamma_{K \ell 3}$ is the $K_{\ell 3}$ decay width, $G_{F}$ is the Fermi constant, $m_{K}$ is the kaon mass, $S_{\mathrm{EW}}$ is the short-distance radiative correction, $\delta_{K}^{\ell}$ is the long-distance radiative correction, $\delta_{\mathrm{SU} 2}$ is the strong isospin-violating effect, $C^{2}$ is 1 for the neutral kaon decay and $1 / 2$ for the charged case, $f_{+}\left(q^{2}\right)$ is the $K^{0} \rightarrow \pi^{-}$vector form factor and $I_{K}^{\ell}$ is the phase-space integral which contains the information of the momentum dependence in the form factors. Averaging over the experimental measurements with appropriate theory inputs of various Standard Model corrections, the product $\left|V_{u s}\right| f_{+}(0)$ is given as [12]

$$
f_{+}(0)\left|V_{u s}\right|=0.2165(4)
$$

with the uncertainty dominated by the experimental measurements and RCs. The form factor $f_{+}(0)$ can be provided by lattice QCD calculations [13-17]. The Flavor Lattice Averaging Group (FLAG) average [18] for $N_{f}=2+1+1$ simulations yields $f_{+}(0)=0.9698(17)$ according to an update on December 2020, which results in a determination of

$$
\left|V_{u s}\right|=0.2232(4)_{\exp +\mathrm{RC}}(4)_{\mathrm{lat}}, \quad \text { for } K_{\ell 3} .
$$

High-precision experimental data on $K_{\mu 2}$ and $\pi_{\mu 2}$ decays $[19,20]$ also accurately determine the ratio $\left|V_{u s} / V_{u d}\right| f_{K^{ \pm}} / f_{\pi^{ \pm}}=0.2760(4) \quad$ [12]. Employing the FLAG $N_{f}=2+1+1$ lattice QCD average [21-24] for the ratio of decay constants $f_{K^{ \pm}} / f_{\pi^{ \pm}}=1.1932(21)$, a value of $\left|V_{u s}\right|=0.2252(5)$ is obtained, which has a $2.6 \sigma$ deviation from the $K_{\ell 3}$-based value. Combining the $\left|V_{u s}\right|$ from $K_{\ell 3}$ and $K_{\mu 2}$ decays yield ${ }^{2}$

$\left|V_{u s}\right|=0.2243(8), \quad$ weighted average of $K_{\ell 3}$ and $K_{\mu 2}$.

\footnotetext{
${ }^{2}$ Here $\left|V_{u s}\right|$ is slightly different from the PDG value $0.2245(8)$ due to the update of the FLAG value of $f_{+}(0)$. Correspondingly, the value of $\left|V_{u d}\right|^{2}+\left|V_{u s}\right|^{2}+\left|V_{u b}\right|^{2}$ given in Eq. (1) also slightly differs from the PDG value of $0.9985(3)_{V_{u d}}(4)_{V_{u s}}$.
}

It should also be mentioned that $\left|V_{u s}\right|$ obtained from hyperon and tau decays are given by $\left|V_{u s}\right|=0.2250$ (27) [25] and 0.2221(13) [26], respectively, both having larger uncertainties than the kaon decays.

To gain a better understanding of the violation of the first-row CKM unitarity in Eq. (1) and the disagreement in the determination of $\left|V_{u s}\right|$ between the $K_{\ell 3}$ and $K_{\mu 2}$, for the $K_{\ell 3}$ decays it requires both a more precise determination of the form factor $f_{+}(0)$ and a direct calculation of RCs from lattice QCD. The latter is more challenging due to the inclusion of both weak and electromagnetic currents in the calculation and is the focus in this paper.

Recently, the horizons of lattice QCD studies have been extended to include various processes with higher-order electroweak interactions. The examples include kaon mixing [27-29], rare kaon decays [30-35], double beta decays [36-44], inclusive $B$-meson decays [45-47], as well as the electromagentic and radiative corrections to the weak decays [48-55]. Among all these processes, the lattice QCD calculation of RCs in $K_{\ell 3}$ still remains one of the largest challenges as it essentially involves a computation of five-point correlation functions. In Ref. [56], it proposes a new method which bridges the lattice QCD calculation with chiral perturbation theory $(C h P T)$ [57,58]. For the $K_{\ell 3}$ decay in the flavor SU(3) limit, it demonstrates that the lattice QCD calculation of the axial $\gamma W$-box diagrams can provide all unknown low-energy constants (LECs) that enter the long-distance radiative correction $\delta_{K}^{\ell}$ in the $C h P T$ representation at the order of $O\left(e^{2} p^{2}\right)$, thus removing the dependence of the RCs on the model used to estimate these LECs. In this paper we will first briefly introduce the methodology and then present the lattice calculation of RCs.

\section{METHODOLOGY}

We start the discussion of the treatment of RCs in $K_{\ell 3}$ decays with two theoretical frameworks: Sirlin's representation and the $C h P T$ representation.

Sirlin's representation is particularly useful in the treatment of the semileptonic decay $H_{i} \rightarrow H_{f} e \bar{\nu}_{e}$ with the hadrons $H_{i}$ and $H_{f}$ having nearly the same masses $m_{i} \approx m_{f}$. In this case, the $O\left(G_{F} \alpha_{e}\right)$ RCs to the decay width is given as [59]
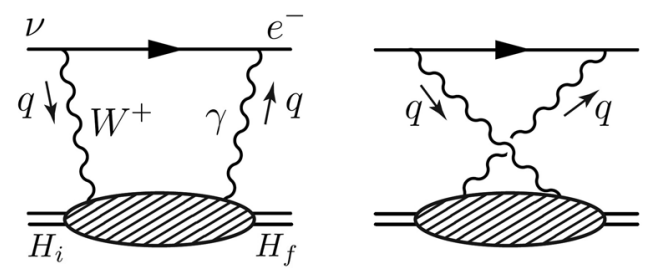

FIG. 1. The $\gamma W$-box diagrams for the semileptonic decay process $H_{i} \rightarrow H_{f} e \bar{\nu}_{e}$. 


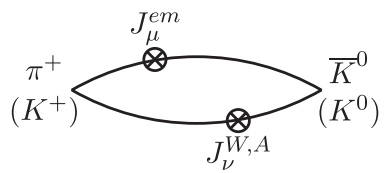

(a)

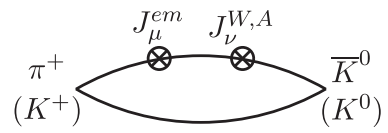

(b)

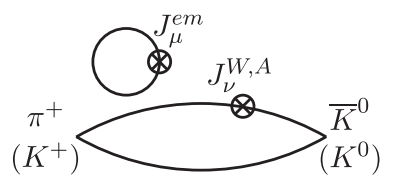

(c)

FIG. 2. Quark contractions for $\bar{K}^{0} \rightarrow \pi^{+} e \bar{\nu}_{e}$ and $K^{0} \rightarrow K^{+} e \bar{\nu}_{e}$.

$\delta=\frac{\alpha_{e}}{2 \pi}\left[\bar{g}+3 \ln \frac{m_{Z}}{m_{p}}+\ln \frac{m_{Z}}{m_{W}}+\tilde{a}_{g}\right]+\delta_{\mathrm{HO}}^{\mathrm{QED}}+2 \square_{\gamma W}^{V A}$,

where $m_{Z}$ and $m_{W}$ are the masses for the $Z$ and $W$ bosons. $m_{p}$ is the proton mass that enters simply by convention. The Sirlin's function $\bar{g}$, which is a function of the electron's end point energy, summarizes the infrared-singular contributions involving both the one-loop and bremsstrahlung corrections [59-61]. The $O\left(\alpha_{s}\right)$ QCD correction $\tilde{a}_{g}$ is dominated by the high-energy scale $Q^{2} \simeq m_{W}^{2}$ with a relatively small contribution of $\frac{\alpha_{e}}{2 \pi} \tilde{a}_{g} \approx-9.6 \times 10^{-5}$ $[59,62]$. The contribution from the resummation of the large QED logs is contained in $\delta_{\mathrm{HO}}^{\mathrm{QED}}=0.0010(3)$ [63]. All the contributions that are sensitive to hadronic scales reside in the axial $\gamma W$-box contribution $\square_{\gamma W}^{V A}$, as shown in Fig. 1. Different from the approach developed in Ref. [64], where an effective field theory is utilized and the $W$ regularization is applied to make the RCs UV finite, here the photon- $W$ box diagram is treated in the full electroweak theory. As all the details were already given by Sirlin in Ref. [59], in this paper we only give a simple illustration in the Appendix to show how $\square_{\gamma W}^{V A}$ is introduced. The total contribution $\delta$ is equivalent to $\left(S_{\mathrm{EW}}-1\right)+\delta_{K}^{e}$ shown in Eq. (2).

In the $K_{\ell 3}$ decays, since $m_{K}$ is not close to $m_{\pi}$, the nonperturbative hadronic effects are contained not only in $\square_{\gamma W}^{V A}$, but also in other diagrams. As a consequence, Eq. (6) cannot be used directly. To evaluate the total RCs, the calculation of the five-point correlation function is required. To simplify this problem, Ref. [56] proposes to calculate the RCs for $\bar{K}^{0} \rightarrow \pi^{+} e \bar{\nu}_{e}$ in the flavor $\operatorname{SU}(3)$ limit, where $m_{K}=m_{\pi}$. The relevant contractions are shown in Fig. 2 with the disconnected diagram (C) vanishing in the flavor SU(3) limit. Although the physical value of $\delta$ cannot be determined directly using this unphysical setup, the lattice calculation can help to extract the LECs for ChPT. Then by using ChPT one can obtain the physical RCs. Besides for the $\bar{K}^{0} \rightarrow \pi^{+}$ transition, the semileptonic decay of $K^{0} \rightarrow K^{+} e \bar{\nu}_{e}$ can also be used to determine the same LECs as it has the same contractions as $\bar{K}^{0} \rightarrow \pi^{+}$up to the disconnected parts.

In $C h P T$, the RCs to $K_{\ell 3}$ are computed to $O\left(e^{2} p^{2}\right)$ $[57,58,65]$ with the short-distance radiative correction

$$
\begin{aligned}
S_{\mathrm{EW}} & =1-e^{2}\left[-\frac{1}{2 \pi^{2}} \ln \frac{M_{Z}}{M_{\rho}}+\left(X_{6}^{\mathrm{phys}}\right)_{\alpha_{s}}\right]+\delta_{\mathrm{HO}}^{\mathrm{QED}} \\
& =1.0229(3),
\end{aligned}
$$

where $M_{\rho}=0.77 \mathrm{GeV}$ is the rho mass and $\left(X_{6}^{\text {phys }}\right)_{\alpha_{s}} \approx$ $3.0 \times 10^{-3}$ [66] summarizes the $O\left(\alpha_{s}\right)$ perturbative QCD (PQCD) contribution to $X_{6}^{\text {phys }}$ with $X_{6}^{\text {phys }}(\mu) \equiv X_{6}^{r}(\mu)-$ $4 K_{12}^{r}(\mu)$ the combination of two renormalized LECs. The scale $\mu$ is usually taken as $\mu=M_{\rho}$ in the numerical analysis. The long-distance radiative correction $\delta_{K}^{\ell}$ has the dependence on the LECs through the relation ${ }^{3}$

$$
\begin{aligned}
& \delta_{K^{ \pm}}^{\ell}=2 e^{2}\left[-\frac{8}{3} X_{1}-\frac{1}{2} \tilde{X}_{6}^{\text {phys }}\left(M_{\rho}\right)\right]+\cdots, \\
& \delta_{K^{0}}^{\ell}=2 e^{2}\left[\frac{4}{3} X_{1}-\frac{1}{2} \tilde{X}_{6}^{\text {phys }}\left(M_{\rho}\right)\right]+\cdots,
\end{aligned}
$$

where the ellipses indicates the omission of the known kinematic terms, which does not depend on the LECs. $X_{1}$ and $\tilde{X}_{6}^{\text {phys }}$ are LECs relevant at $O\left(e^{2} p^{2}\right) . \tilde{X}_{6}^{\text {phys }}\left(M_{\rho}\right) \equiv$ $X_{6}^{\text {phys }}\left(M_{\rho}\right)+\left(2 \pi^{2}\right)^{-1} \ln \left(M_{Z} / M_{\rho}\right)-\left(X_{6}^{\text {phys }}\right)_{\alpha_{s}}$ removes the large electroweak logarithm and the $O\left(\alpha_{s}\right)$ PQCD correction from $X_{6}^{\text {phys }}$. In a similar way, one can define the quantity $\delta_{\pi^{ \pm}}^{\ell}$ for $\pi_{\ell 3}$

$$
\delta_{\pi^{ \pm}}^{\ell}=2 e^{2}\left[-\frac{2}{3} X_{1}-\frac{1}{2} \tilde{X}_{6}^{\mathrm{phys}}\left(M_{\rho}\right)\right]+\cdots
$$

Since the neutral kaon decay mode $\bar{K}^{0} \rightarrow \pi^{+} e \bar{\nu}_{e}$ is theoretically cleaner as it does not receive contributions from the $\pi^{0}-\eta$ mixing, which complicates the analysis in the flavor SU(3) limit, we may use it to extract the LECs. Comparing the ChPT and Sirlin's representations, the relation between the axial $\gamma W$-box contribution $\left.\square_{\gamma W}^{V A}\right|_{K^{0}, \mathrm{SU}(3)}$ and the LECs is given by [56]

\footnotetext{
${ }^{3}$ Notice that in a similar expression in Ref. [56], the quantity $\delta_{K^{ \pm}}^{\ell}$ includes also contributions from the LECs $\left\{K_{i}^{r}\right\}$. That, however, was not the standard convention adopted by the ChPT community, which chooses to lump the $\left\{K_{i}^{r}\right\}$ contribution into $\delta_{\mathrm{SU} 2}$
} 
TABLE I. Ensembles used in this work. For each ensemble we list the pion mass $m_{\pi}$, the spatial and temporal extents, $L$ and $T$, the inverse of lattice spacing $a^{-1}$, the number of configurations used, $N_{\text {conf }}$, and the minimal time separation between the kaon/pion operators and the current insertions, $\Delta t$.

\begin{tabular}{lccccccc}
\hline \hline Ensemble & & $m_{\pi}[\mathrm{MeV}]$ & $L$ & $T$ & $a^{-1}[\mathrm{GeV}]$ & $N_{\text {conf }}$ & $\Delta t[\mathrm{fm}]$ \\
\hline DSDR & 24D & $141.2(4)$ & 24 & 64 & 1.015 & 46 \\
& 32D & $141.4(3)$ & 32 & 64 & 1.015 & 32 \\
Iwasaki & 32D-fine & $143.0(3)$ & 32 & 64 & 1.378 & 71 \\
& 48I & $135.5(4)$ & 48 & 96 & 1.730 & 1.56 \\
& 64I & $135.3(2)$ & 64 & 128 & 2.359 & 62 & 1.43 \\
\hline \hline
\end{tabular}

$$
\begin{aligned}
-\frac{8}{3} X_{1}+\bar{X}_{6}^{\text {phys }}\left(M_{\rho}\right)= & -\frac{1}{2 \pi \alpha}\left(\left.\square_{\gamma W}^{V A}\right|_{K^{0}, \mathrm{SU}(3)}-\frac{\alpha}{8 \pi} \ln \frac{M_{W}^{2}}{M_{\rho}^{2}}\right) \\
& +\frac{1}{8 \pi^{2}}\left(\frac{5}{4}-\tilde{a}_{g}\right),
\end{aligned}
$$

with $\quad \bar{X}_{6}^{\text {phys }}\left(M_{\rho}\right) \quad$ defined as $\quad \bar{X}_{6}^{\text {phys }}\left(M_{\rho}\right) \equiv X_{6}^{\text {phys }}\left(M_{\rho}\right)+$ $\left(2 \pi^{2}\right)^{-1} \ln \left(M_{Z} / M_{\rho}\right)$, which removes only the large electroweak logarithm but retains the full PQCD corrections. For the $\pi_{\ell 3}$ decay, the relation is given by

$$
\begin{aligned}
& \frac{4}{3} X_{1}+\bar{X}_{6}^{\text {phys }}\left(M_{\rho}\right) \\
& \quad=-\frac{1}{2 \pi \alpha}\left(\left.\square_{\gamma W}^{V A}\right|_{\pi}-\frac{\alpha}{8 \pi} \ln \frac{M_{W}^{2}}{M_{\rho}^{2}}\right)+\frac{1}{8 \pi^{2}}\left(\frac{5}{4}-\tilde{a}_{g}\right)
\end{aligned}
$$

The box contribution $\left.\square_{\gamma W}^{V A}\right|_{\pi}$ for the $\pi_{\ell 3}$ decay has been calculated in Ref. [8]. The focus of this paper is on the determination of $\left.\square_{\gamma W}^{V A}\right|_{K^{0}, \mathrm{SU}(3)}$, from which the LECs $X_{1}$ and $\bar{X}_{6}^{\text {phys }}\left(M_{\rho}\right)$ can be obtained.

The lattice QCD calculation of $\left.\square_{\gamma W}^{V A}\right|_{K^{0}, \mathrm{SU}(3)}$ can follow the procedures given in Ref. [8]. We first define the hadronic function $\mathcal{H}_{\mu \nu}^{V A}(t, \vec{x})$ in Euclidean space

$$
\mathcal{H}_{\mu \nu}^{V A}(t, \vec{x}) \equiv\left\langle\pi^{+}(P)\left|T\left[J_{\mu}^{\mathrm{em}}(t, \vec{x}) J_{\nu}^{W, A}(0)\right]\right| \bar{K}^{0}(P)\right\rangle,
$$

where $J_{\mu}^{\mathrm{em}}=\frac{2}{3} \bar{u} \gamma_{\mu} u-\frac{1}{3} \bar{d} \gamma_{\mu} d-\frac{1}{3} \bar{s} \gamma_{\mu} s$ is the electromagnetic quark current, and $J_{\nu}^{W, A}=\bar{u} \gamma_{\nu} \gamma_{5} s$ is the axial part of the weak charged current. The Euclidean momentum $P$ is chosen as $P=\left(i m_{K}, \overrightarrow{0}\right)$ with $m_{K}=m_{\pi}$ in the flavor $\mathrm{SU}(3)$ limit. The box contribution $\left.\square_{\gamma W}^{V A}\right|_{K^{0}, \mathrm{SU}(3)}$ can be determined through the integral

$$
\left.\square_{\gamma W}^{V A}\right|_{K^{0}, \mathrm{SU}(3)}=\frac{3 \alpha_{e}}{2 \pi} \int \frac{d Q^{2}}{Q^{2}} \frac{m_{W}^{2}}{m_{W}^{2}+Q^{2}} M_{K}\left(Q^{2}\right)
$$

with

$$
\begin{aligned}
M_{K}\left(Q^{2}\right) & =-\frac{1}{6} \frac{\sqrt{Q^{2}}}{m_{K}} \int d^{4} x \omega(t, \vec{x}) \epsilon_{\mu \nu \alpha 0} x_{\alpha} \mathcal{H}_{\mu \nu}^{V A}(t, \vec{x}), \\
\omega(t, \vec{x}) & =\int_{-\frac{\pi}{2}}^{\frac{\pi}{2}} \frac{\cos ^{3} \theta d \theta}{\pi} \frac{j_{1}\left(\sqrt{Q^{2}}|\vec{x}| \cos \theta\right)}{|\vec{x}|} \cos \left(\sqrt{Q^{2}} t \sin \theta\right) .
\end{aligned}
$$

Here $j_{1}(x)$ is the spherical Bessel function. To compute the integral in Eq. (13), for small $Q^{2}$, we use lattice QCD input of $\mathcal{H}_{\mu \nu}^{V A}(t, \vec{x})$. The operator product $J_{\mu}^{\mathrm{em}}(x) J_{\nu}^{W}(0)$ behaves as $\epsilon_{\mu \nu \alpha \beta}\left(x_{\alpha} / x^{4}\right) J_{\beta}^{W, V}(0)$ when $x \rightarrow 0$. Thus the integral of $M_{K}\left(Q^{2}\right)$ is well behaved at small $Q^{2}$. The situation is different from the lattice calculation of the hadronic vacuum polarization function where $J_{\mu}^{\mathrm{em}}(x) J_{\nu}^{\mathrm{em}}(0)$ behaves as $\left(g_{\mu \nu} \partial^{2}-\partial_{\mu} \partial_{\nu}\right) / x^{4}$ when $x \rightarrow 0$. For large $Q^{2}$, the operator product expansion of $J_{\mu}^{\mathrm{em}}(x) J_{\nu}^{W, A}(0)$ is utilized with the Wilson coefficients given at the four-loop accuracy $[67,68]$. For more details, we refer the readers to Ref. [8].

\section{NUMERICAL RESULTS}

Five gauge ensemble with $N_{f}=2+1$-flavor domain wall fermion are used in the calculation. The detailed information is shown in Table I. Here 48I and 64I use the Iwasaki gauge action in the simulation (denoted as Iwasaki) while the other three ensembles use Iwasaki + DSDR action (denoted as dislocation suppressing determinant ratio (DSDR)). We place the Coulomb gauge-fixed wall-source quark propagators on all time slices. For each configuration we calculate pointsource propagators at 1024 (2048 for 32D) random spacetime locations. This setup allows us to perform $O(1000)$ measurements per configuration. The number of configurations for each ensemble is listed in Table I. We use wall-source operators to create the kaon in the initial state and the pion in the final states. The wall-source operators are known to have a good overlap with the meson ground state. Using $\Delta t$ to specify the minimal time separation between meson interpolating operators and the current insertions, we find the ground-state saturation for $\Delta t \sim 1 \mathrm{fm}$ in our past studies of various kaon/pion related quantities. In this work, to be conservative, we chose $\Delta t$ with a range of about 1.4-1.5 fm as shown in Table I and the excited-state contamination can 

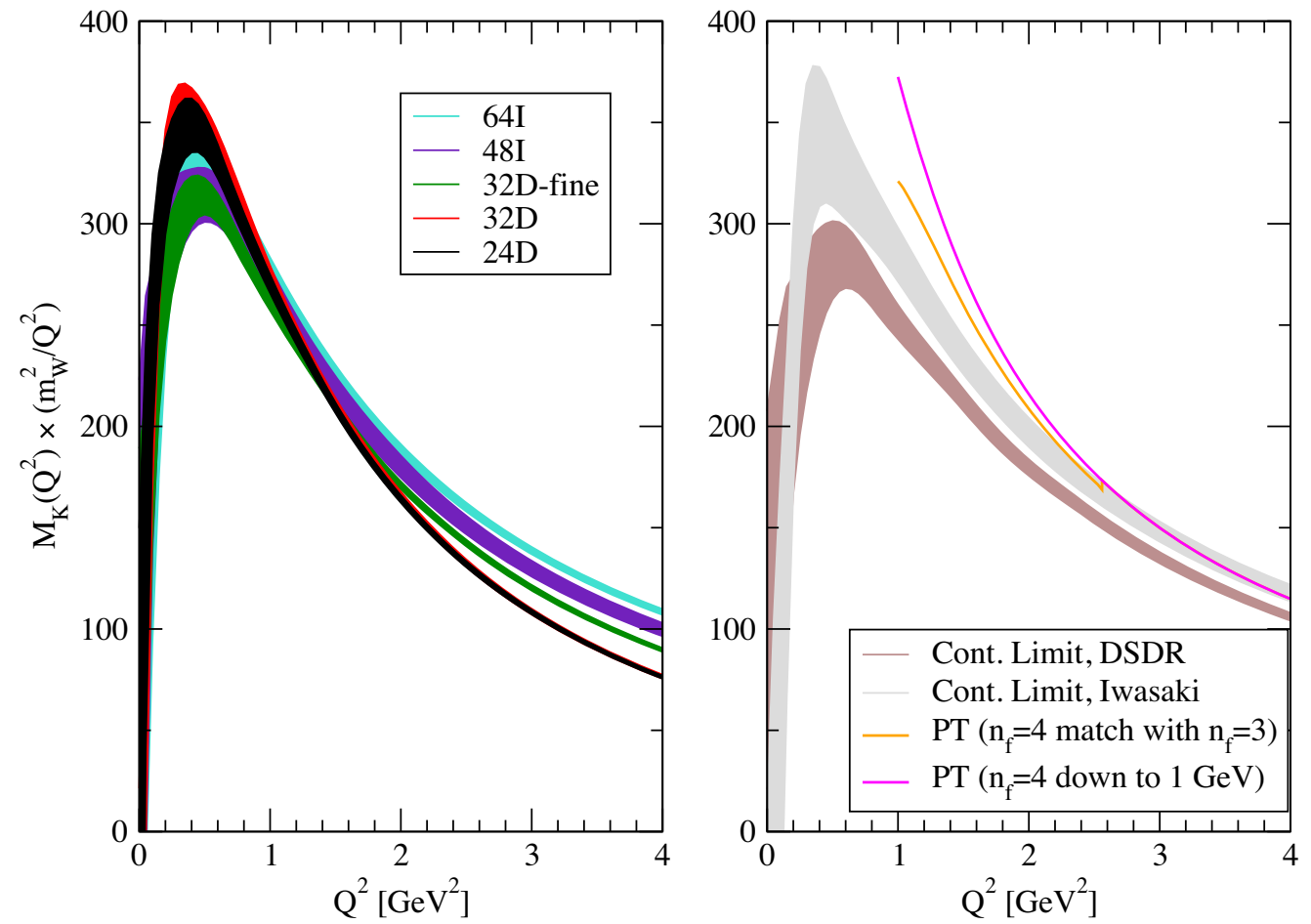

FIG. 3. $M_{K}\left(Q^{2}\right) \times\left(m_{W}^{2} / Q^{2}\right)$ as a function of $Q^{2}$. In the left panel, the lattice results for all five ensembles are given. In the right panel, we have extrapolated the Iwasaki and DSDR results to their continuum limit. The remaining orange and magenta curves are the results from perturbation theory.

be safely neglected. We use local vector and axial-vector currents in the calculation. These currents are matched to the conserved ones by multiplying the renormalization factors $Z_{V / A}$, whose value are quoted from Ref. [69]. The correlation functions are constructed using the field sparsening technique $[70,71]$ with a significant reduction in the propagator storage.

For the locations of two current insertions $J_{\mu}^{\mathrm{em}}$ and $J_{\nu}^{W, A}$, we treat one as the source of the propagator and the other as the sink. In this way the hadronic function $\mathcal{H}_{\mu \nu}^{V A}(t, \vec{x})$, which depends on the coordinate-space variable $x$ can be obtained. Such technique has also been used in the computation of three-point correction function to extract the pion charge radius [72]. The flavor $\mathrm{SU}(3)$ limit is achieved by tuning down the strange quark mass to be the same as the light quark mass.
Inserting $\mathcal{H}_{\mu \nu}^{V A}(t, \vec{x})$ into the integral (14), we calculate the scalar function $M_{K}\left(Q^{2}\right)$. The lattice results for $M_{K}\left(Q^{2}\right) \times$ $\left(m_{W}^{2} / Q^{2}\right)$ as a function of $Q^{2}$ are shown in the left panel of Fig. 3. At large $Q^{2}\left(Q^{2} \gtrsim 1 \mathrm{GeV}^{2}\right)$, the lattice results from different gauge ensembles start to disagree, suggesting the obvious lattice discretization effects. In the right panel of Fig. 3, a continuum extrapolation is performed to obtain the results in the continuum limit for Iwasaki and DSDR ensembles separately. To reduce the systematic uncertainties contained in the lattice data at large $Q^{2}$, we calculate the $M_{K}\left(Q^{2}\right)$ in PQCD using the RunDec package [73]. At low $Q^{2}$ the perturbative results suffer from large PQCD truncation effects due to the lack of higher-loop and higher-twist contributions. We observe an expected discrepancy between the orange and magenta curves at low $Q^{2}$, where the former

TABLE II. For each ensemble, given three choices of $Q_{\text {cut }}^{2}$, the lattice results of $\left.\square_{\gamma W}^{V A, \leq}\right|_{K^{0}, \mathrm{SU}(3)}$ are shown.

\begin{tabular}{|c|c|c|c|c|}
\hline \multicolumn{5}{|c|}{$\left.\square_{\gamma W}^{V A, \leq}\right|_{K^{0}, \mathrm{SU}(3)}$} \\
\hline \multicolumn{5}{|c|}{ Ensemble } \\
\hline \multirow[t]{2}{*}{$Q_{\text {cut }}^{2}$} & & $1 \mathrm{GeV}^{2}$ & $2 \mathrm{GeV}^{2}$ & $3 \mathrm{GeV}^{2}$ \\
\hline & $24 \mathrm{D}$ & $0.160(6) \times 10^{-3}$ & $0.274(7) \times 10^{-3}$ & $0.346(7) \times 10^{-3}$ \\
\hline \multirow[t]{2}{*}{ DSDR } & 32D & $0.160(5) \times 10^{-3}$ & $0.275(5) \times 10^{-3}$ & $0.347(6) \times 10^{-3}$ \\
\hline & 32D-fine & $0.145(6) \times 10^{-3}$ & $0.260(6) \times 10^{-3}$ & $0.337(7) \times 10^{-3}$ \\
\hline \multirow[t]{2}{*}{ Iwasaki } & $48 \mathrm{I}$ & $0.149(8) \times 10^{-3}$ & $0.268(8) \times 10^{-3}$ & $0.350(9) \times 10^{-3}$ \\
\hline & $64 \mathrm{I}$ & $0.149(7) \times 10^{-3}$ & $0.273(8) \times 10^{-3}$ & $0.360(9) \times 10^{-3}$ \\
\hline
\end{tabular}




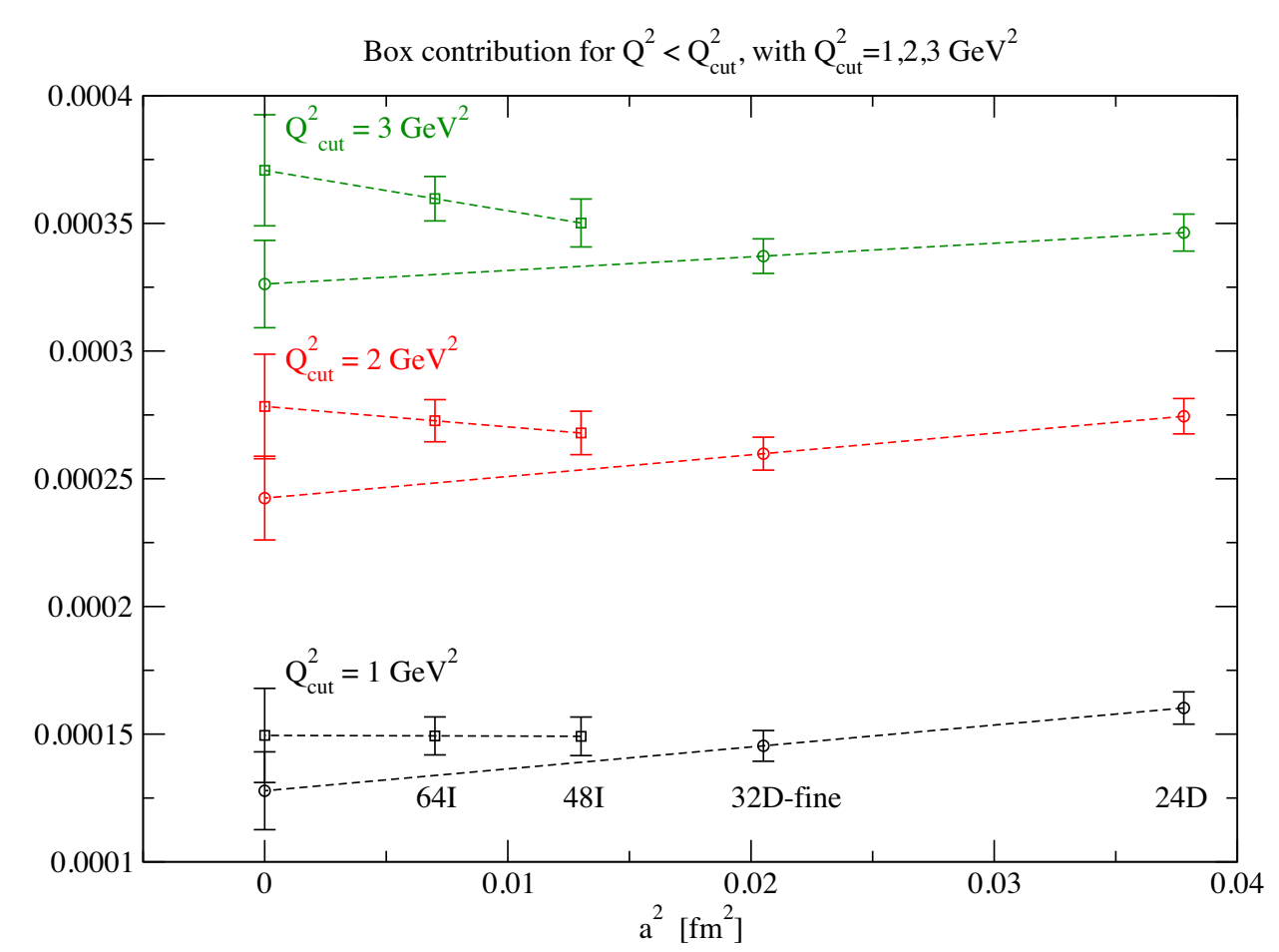

FIG. 4. For three choices of $Q_{\text {cut }}^{2}$, the continuum extrapolation of $\left.\square_{\gamma W}^{V A, \leq}\right|_{K^{0}, \mathrm{SU}(3)}$ is performed for Iwasaki and DSDR ensembles, respectively.

uses the 4-flavor theory down to $1 \mathrm{GeV}$, while the latter turns to the 3-flavor theory upon decoupling the charm quark at $1.6 \mathrm{GeV}$.

We introduce a momentum-squared scale $Q_{\text {cut }}^{2}$ that separates the $Q^{2}$ integral into two regimes. We use the lattice data to determine the integral for $Q^{2} \leq Q_{\text {cut }}^{2}$ and perturbation theory to determine the integral for $Q^{2}>Q_{\text {cut }}^{2}$. Three values of $Q_{\text {cut }}^{2}=1,2,3 \mathrm{GeV}^{2}$ are used to check the
$Q_{\text {cut }}^{2}$ dependence in the final results. The lattice results of $\left.\square_{\gamma W}^{V A, \leq}\right|_{K^{0}, \mathrm{SU}(3)}$ for each ensemble are listed in Table II and the continuum extrapolation is shown in Fig. 4. After the extrapolation, the lattice results for $\left.\square_{\gamma W}^{V A, \leq}\right|_{K^{0}, \mathrm{SU}(3)}$ and perturbative results for $\left.\square_{\gamma W}^{V A,>}\right|_{K^{0}, \mathrm{SU}(3)}$ are listed in Table III.

Combining the lattice data and perturbative results given in Table III, we have

$$
\left.\square_{\gamma W}^{V A}\right|_{K^{0}, \mathrm{SU}(3)}= \begin{cases}2.460(18)_{\mathrm{stat}}(42)_{\mathrm{PT}}(22)_{a}(1)_{\mathrm{FV}} \times 10^{-3} & Q_{\mathrm{cut}}^{2}=1 \mathrm{GeV}^{2} \\ 2.437(20)_{\mathrm{stat}}(15)_{\mathrm{PT}}(36)_{a}(1)_{\mathrm{FV}} \times 10^{-3} & Q_{\mathrm{cut}}^{2}=2 \mathrm{GeV}^{2} \\ 2.433(22)_{\mathrm{stat}}(07)_{\mathrm{PT}}(45)_{a}(1)_{\mathrm{FV}} \times 10^{-3} & Q_{\mathrm{cut}}^{2}=3 \mathrm{GeV}^{2}\end{cases}
$$

TABLE III. Using the scale $Q_{\text {cut }}^{2}$ to split the integral range, the contributions of $\left.\square_{\gamma W}^{V A, \leq}\right|_{K^{0}, \mathrm{SU}(3)}$ from lattice QCD and $\left.\square_{\gamma W}^{V A,>}\right|_{K^{0}, \mathrm{SU}(3)}$ from perturbation theory are shown. For the lattice results, we have performed the continuum extrapolation for Iwasaki and DSDR ensembles as shown in Fig. 4. Here we show the results after the extrapolation. For the perturbative results, the central values are compiled using the 4-flavor theory and uncertainties include the higher-loop effects and the higher-twist effects with the error analysis following Ref. [8].

\begin{tabular}{lccc}
\hline \hline & \multicolumn{3}{c}{} \\
\cline { 2 - 4 }$Q_{\text {cut }}^{2}$ & $\left.\square_{\gamma W}^{V A, \leq}\right|_{K^{0}, \mathrm{SU}(3)}$ & DSDR & $\left.\square_{\gamma W}^{V A,>}\right|_{K^{0}, \mathrm{SU}(3)}$ \\
\hline $1 \mathrm{GeV}^{2}$ & Iwasaki & $0.128(15) \times 10^{-3}$ & $\mathrm{PQCD}$ \\
$2 \mathrm{GeV}^{2}$ & $0.150(18) \times 10^{-3}$ & $0.242(16) \times 10^{-3}$ & $2.310(42) \times 10^{-3}$ \\
$3 \mathrm{GeV}^{2}$ & $0.278(20) \times 10^{-3}$ & $0.326(17) \times 10^{-3}$ & $2.159(15) \times 10^{-3}$ \\
\hline \hline
\end{tabular}


Here we take the combination of the Iwasaki and perturbative results as the central value and estimate the residual lattice artifacts (with a subscript $a$ ) using the discrepancy between Iwasaki and DSDR. The lattice finite-volume effects (with a subscript FV) are estimated by comparing the $24 \mathrm{D}$ and $32 \mathrm{D}$ results. As a final result, we quote the value of $\left.\square_{\gamma W}^{V A}\right|_{K^{0}, \mathrm{SU}(3)}$ at $Q_{\mathrm{cut}}^{2}=2 \mathrm{GeV}^{2}$ and add the statistical and systematic errors in quadrature

$$
\left.\square_{\gamma W}^{V A}\right|_{K^{0}, \mathrm{SU}(3)}=2.437(44) \times 10^{-3} .
$$

Inserting the result of $\left.\square_{\gamma W}^{V A}\right|_{K^{0}, \mathrm{SU}(3)}$ into Eq. (10), we obtain

$$
\begin{aligned}
-\frac{8}{3} X_{1}+\bar{X}_{6}^{\text {phys }} & =22.6(1.0) \times 10^{-3} \text { or } \\
-\frac{8}{3} X_{1}+\tilde{X}_{6}^{\text {phys }} & =19.6(1.0) \times 10^{-3} .
\end{aligned}
$$

The previous ChPT analysis [58] quoted the LECs from the minimal resonance model $[66,74]$ with

$$
X_{1}=-3.7(3.7) \times 10^{-3}, \quad \tilde{X}_{6}^{\text {phys }}=10.4(10.4) \times 10^{-3} .
$$

As it is hard to accurately estimate the uncertainty in these LECs from the ChPT perspective, Ref. [58] attributed to them a $100 \%$ uncertainty. Combining $X_{1}$ and $\tilde{X}_{6}^{\text {phys }}$ together yields

$$
\begin{aligned}
& -\frac{8}{3} X_{1}+\tilde{X}_{6}^{\text {phys }} \\
& =20.3(14.3) \times 10^{-3} \quad[\text { minimal resonance model }] .
\end{aligned}
$$

Our result for $-\frac{8}{3} X_{1}+\tilde{X}_{6}^{\text {phys }}$ agrees with the minimal resonance model within few percent. Such a good agreement could easily be fortuitous as the methods used in the two studies are very different and a large uncertainty is assigned to the estimate based on the model.

For the $\pi_{\ell 3}$ decay, substituting the lattice QCD result $\left.\square_{\gamma W}^{V A}\right|_{\pi}=2.830(28) \times 10^{-3}$ [8] into Eq. (11) yields

$$
\frac{4}{3} X_{1}+\bar{X}_{6}^{\text {phys }}=14.0(6) \times 10^{-3} .
$$

Combining Eqs. (17) and (20) together, we have

$X_{1}=-2.2(4) \times 10^{-3}, \quad \bar{X}_{6}^{\text {phys }}=16.9(7) \times 10^{-3}$.

Here the uncertainty is estimated conservatively through a linear addition. It should be pointed out that in Eq. (21) the estimate of the higher order terms in the ChPT expansion are not included yet.

In $C h P T$, the RCs $\delta_{K}^{\ell}$ have two major sources of theoretical uncertainties: the input of the LECs at
$O\left(e^{2} p^{2}\right)$ and the unknown $O\left(e^{2} p^{4}\right)$ terms in the ChPT expansion. Using the LECs from this calculation, the former uncertainty is significantly reduced, while the latter one remains. It results in an update of $\delta_{K}^{\ell}$ (in units of \%)

$$
\begin{aligned}
\delta_{K^{0}}^{e} & =0.99(19)_{e^{2} p^{4}}(11)_{\mathrm{LEC}} \rightarrow 1.00(19), \\
\delta_{K^{0}}^{\mu} & =1.40(19)_{e^{2} p^{4}}(11)_{\mathrm{LEC}} \rightarrow 1.41(19), \\
\delta_{K^{ \pm}}^{e} & =0.10(19)_{e^{2} p^{4}}(16)_{\mathrm{LEC}} \rightarrow-0.01(19), \\
\delta_{K^{ \pm}}^{\mu} & =0.02(19)_{e^{2} p^{4}}(16)_{\mathrm{LEC}} \rightarrow-0.09(19) .
\end{aligned}
$$

We refrain from presenting a corresponding update of $\left|V_{u s}\right|$ in this paper, because (1) our results for $\delta_{K}^{\ell}$ still agree with the existing literature within error bars, and (2) our lattice calculation removes only the LEC uncertainty but not the dominant $\mathcal{O}\left(e^{2} p^{4}\right)$ uncertainty. Therefore, we shall instead await a next round of global analysis in the near future such as that in Ref. [11], whose impact on the precision lowenergy tests will be more significant. Our lattice result may serve as an important input to such an analysis.

\section{CONCLUSION}

Modern-day lattice QCD has reached the era when realistic calculations for many interesting second-order electroweak processes have become feasible. In this work we perform a study of the $\gamma W$-box correction to the kaon semileptonic decay $\bar{K}^{0} \rightarrow \pi^{+} e \bar{\nu}_{e}$. We adopt the new method proposed in Ref. [56], which connects the Sirlin's representation to the $C h P T$ representation in the flavor $\mathrm{SU}(3)$ limit. It allows us to determine the LECs for ChPT by computing the axial $\gamma W$-box correction. We find that the values of the LECs devised from the lattice calculation agree well with the minimal resonance model used in the literature, while a dramatic reduction of the respective uncertainties is achieved. From Table III, we find that the nonperturbative lattice QCD contribution amounts to about $10 \%$ of $\left.\square_{\gamma W}^{V A}\right|_{K^{0}, \mathrm{SU}(3)}$, while the remaining contribution is from the perturbative part. Thus even a $10 \%$ precision in the nonperturbative contribution is enough to guarantee a percent-level accuracy of the determination of the box diagram and, consequently of the relevant LECs. Finally, these LECs are used to estimate the RCs $\delta_{K}^{\ell}$ and help to reduce its uncertainty. To further improve the determination of RCs, the inclusion of higher-order terms in $C h P T$ and the lattice QCD computation of the complete set of Feynman diagrams are necessary.

\section{ACKNOWLEDGMENTS}

X. F. and L. C. J. gratefully acknowledge many helpful discussions with our colleagues from the RBC-UKQCD Collaborations. X. F. and P. X. M. were supported in part by NSFC of China under Grants No. 11775002 and No. 12070131001 and National Key Research and 
Development Program of China under Contracts No. 2020YFA0406400. M. G. is supported by EU Horizon 2020 research and innovation programme, STRONG-2020 project, under Grant Agreement No. 824093 and by the German-Mexican research collaboration Grants No. 278017 (CONACyT) and No. SP 778/4-1 (DFG). L. C. J. acknowledges support by DOE Office of Science Early Career Award No. DE-SC0021147 and DOE Grant No. DE-SC0010339. The work of C. Y. S. is supported in part by the DFG (Project-ID 196253076-TRR 110) and the NSFC (Grant No. 11621131001) through the funds provided to the Sino-German CRC 110 "Symmetries and the Emergence of Structure in QCD," and also by the Alexander von Humboldt Foundation through the Humboldt Research Fellowship. The computation is performed under the ALCC Program of the US DOE on the Blue Gene/Q (BG/Q) Mira computer at the Argonne Leadership Class Facility, a DOE Office of Science Facility supported under Contract No. DEAC02-06CH11357. Computations for this work were carried out in part on facilities of the USQCD Collaboration, which are funded by the Office of Science of the U.S. Department of Energy. The calculation is also carried out on Tianhe 3 prototype at Chinese National Supercomputer Center in Tianjin.

\section{APPENDIX: $\gamma \boldsymbol{w}$-BOX DIAGRAM CONTRIBUTION FROM FULL ELECTROWEAK THEORY}

The $\gamma W$-box diagram contribution to the $K(p) \rightarrow$ $\pi\left(p^{\prime}\right) e\left(p_{e}\right) \bar{\nu}\left(p_{\nu}\right)$ process is given by the amplitude

$$
\begin{aligned}
M_{\gamma W}= & \frac{g^{2} e^{2}}{8} \int \frac{d^{4} q}{(2 \pi)^{4}} \frac{1}{\left(q+p^{\prime}-p\right)^{2}-M_{W}^{2}} \\
& \times \frac{\bar{u}_{e} \gamma^{\mu}\left(\not p_{e}-\not 1+m_{e}\right) \gamma^{\nu}\left(1-\gamma_{5}\right) v_{\nu}}{\left[\left(p_{e}-q\right)^{2}-m_{e}^{2}\right]\left[q^{2}-M_{\gamma}^{2}\right]} T_{\mu \nu}\left(q ; p^{\prime}, p\right),
\end{aligned}
$$

where

$T_{\mu \nu}\left(q ; p^{\prime}, p\right)=\int d^{4} x e^{i q \cdot x}\left\langle\pi\left(p^{\prime}\right)\left|T\left\{J_{\mu}^{\mathrm{em}}(x) J_{\nu}^{W}(0)\right\}\right| K(p)\right\rangle$.

Since $M_{W} \gg p^{\prime}-p$, one can set $1 /\left(\left(q+p^{\prime}-p\right)^{2}-M_{W}^{2}\right) \rightarrow$ $1 /\left(q^{2}-M_{W}^{2}\right)$ where the induced error is of order $1 / M_{W}^{2}$ which is negligible. Furthermore, using $g^{2} /\left(8 M_{W}^{2}\right)=$ $G_{F} / \sqrt{2}$, one obtains:

$$
\begin{aligned}
M_{\gamma W}= & -\frac{G_{F} e^{2}}{\sqrt{2}} \int \frac{d^{4} q}{(2 \pi)^{4}} \frac{M_{W}^{2}}{M_{W}^{2}-q^{2}} \\
& \times \frac{\bar{u}_{e} \gamma^{\mu}\left(\not p_{e}-\not 1+m_{e}\right) \gamma^{\nu}\left(1-\gamma_{5}\right) v_{\nu}}{\left[\left(p_{e}-q\right)^{2}-m_{e}^{2}\right]\left[q^{2}-M_{\gamma}^{2}\right]} T_{\mu \nu}\left(q ; p^{\prime}, p\right) .
\end{aligned}
$$

This integral is UV finite because of the existence of the full $W$ propagator. So there is no need to introduce any extra UV regularization.

Next, the lepton spinor can be split into several pieces using the identity:

$$
\gamma^{\mu} \gamma^{\nu} \gamma^{\rho}=g^{\mu \nu} \gamma^{\rho}-g^{\mu \rho} \gamma^{\nu}+g^{\nu \rho} \gamma^{\mu}-i \epsilon^{\mu \nu \rho \alpha} \gamma_{\alpha} \gamma_{5} .
$$

In this project we work in the limit $M_{K} \rightarrow M_{\pi}$. In this limit, according to Sirlin's current algebra analysis [59], the only piece in $M_{\gamma W}$ that depends on the details of nonperturbative QCD is the term with an $\epsilon$-tensor from the lepton spinor. This term is IR finite, so one can set $M_{\gamma}=0, p_{e}=0$ and $p=p^{\prime}=P$ in the integral. Also this term can only survive if there is an $\epsilon$ tensor from $T_{\mu \nu}$, which is contributed from the axial component of the charged weak current. One can then write this term as

$$
M_{\gamma W}^{\epsilon}=-\frac{G_{F}}{\sqrt{2}} \bar{u}_{e} \gamma_{\lambda}\left(1-\gamma_{5}\right) v_{\nu} I^{\lambda}
$$

where

$$
I^{\lambda}=i e^{2} \int \frac{d^{4} q}{(2 \pi)^{4}} \frac{M_{W}^{2}}{M_{W}^{2}-q^{2}} \frac{\epsilon^{\mu \nu \alpha \lambda} q_{\alpha}}{\left(q^{2}\right)^{2}} T_{\mu \nu}(q ; P, P) .
$$

The effect of $I^{\lambda}$ can be expressed as a relative correction to the hadron form factor:

$$
I^{\lambda}=\left\langle\pi(P)\left|J_{W}^{\lambda}(0)\right| K(P)\right\rangle \square_{\gamma W}^{V A}=2 F_{+} \square_{\gamma W}^{V A} P^{\lambda},
$$

where $P^{2}=M^{2}$ gives the squared mass of the degenerate meson. That gives

$\square_{\gamma W}^{V A}=\frac{i e^{2}}{2 M^{2}} \int \frac{d^{4} q}{(2 \pi)^{4}} \frac{M_{W}^{2}}{M_{W}^{2}-q^{2}} \frac{\epsilon^{\mu \nu \alpha \lambda} q_{\alpha} P_{\lambda}}{\left(q^{2}\right)^{2}} \frac{T_{\mu \nu}(q ; P, P)}{F_{+}}$.

Therefore, $\square_{\gamma W}^{V A}$ is a UV- and IR-finite quantity. The above derivations have also been presented in Ref. [56]. In Euclidean space, following the procedures given in Ref. [8], one can write the expression of $\square_{\gamma W}^{V A}$ in the form of Eq. (13).

Although $\square_{\gamma W}^{V A}$ is UV finite, there are other diagrams in the $\mathcal{O}\left(\alpha_{\mathrm{em}}\right)$ RCs to the hadron semileptonic beta decays that are UV divergent in the full electroweak theory. The examples are the wave function renormalization and vertex corrections. 
In those contributions, one needs to split the photon propagator into two pieces following Sirlin's approach,

$$
\frac{1}{q^{2}}=\frac{1}{q^{2}-M_{W}^{2}}+\frac{M_{W}^{2}}{M_{W}^{2}-q^{2}} \frac{1}{q^{2}}
$$

in order to separate the UV and IR physics. The first term on the right-hand side gives UV-divergent integrals. However, it was shown by Sirlin in Ref. [59] that this divergent structure is exactly the same as in the $\mathcal{O}\left(\alpha_{\mathrm{em}}\right) \mathrm{RCs}$ in the muon decay. Therefore, as long as one defines the Fermi's constant $G_{F}$ as that measured from the muon decay, all the $\mathcal{O}\left(\alpha_{\mathrm{em}}\right)$ RCs to semileptonic beta decay processes are completely UV finite in the full electroweak theory. As a result, there is no renormalization scale in Sirlin's representation (6).
[1] P. A. Zyla et al. (Particle Data Group), Prog. Theor. Exp. Phys. 2020, 083C01 (2020).

[2] J. C. Hardy and I. S. Towner, Phys. Rev. C 91, 025501 (2015).

[3] J. C. Hardy and I. S. Towner, Phys. Rev. C 102, 045501 (2020).

[4] C. Y. Seng, M. Gorchtein, and M. J. Ramsey-Musolf, Phys. Rev. D 100, 013001 (2019).

[5] M. Gorchtein, Phys. Rev. Lett. 123, 042503 (2019).

[6] C.-Y. Seng, M. Gorchtein, H. H. Patel, and M. J. RamseyMusolf, Phys. Rev. Lett. 121, 241804 (2018).

[7] M. Tanabashi et al. (Particle Data Group), Phys. Rev. D 98, 030001 (2018).

[8] X. Feng, M. Gorchtein, L.-C. Jin, P.-X. Ma, and C.-Y. Seng, Phys. Rev. Lett. 124, 192002 (2020).

[9] C.-Y. Seng, X. Feng, M. Gorchtein, and L.-C. Jin, Phys. Rev. D 101, 111301 (2020).

[10] C.-Y. Seng and U.-G. Meißner, Phys. Rev. Lett. 122, 211802 (2019).

[11] M. Antonelli et al. (FlaviaNet Working Group on Kaon Decays), Eur. Phys. J. C 69, 399 (2010).

[12] M. Moulson, Proc. Sci., CKM2016 (2017) 033 [arXiv:1704.04104].

[13] P. A. Boyle et al. (RBC/UKQCD Collaborations), J. High Energy Phys. 06 (2015) 164.

[14] N. Carrasco, P. Lami, V. Lubicz, L. Riggio, S. Simula, and C. Tarantino, Phys. Rev. D 93, 114512 (2016).

[15] S. Aoki, G. Cossu, X. Feng, H. Fukaya, S. Hashimoto, T. Kaneko, J. Noaki, and T. Onogi (JLQCD Collaboration), Phys. Rev. D 96, 034501 (2017).

[16] A. Bazavov et al. (Fermilab Lattice and MILC Collaborations), Phys. Rev. D 99, 114509 (2019).

[17] J. Kakazu, K.-i. Ishikawa, N. Ishizuka, Y. Kuramashi, Y. Nakamura, Y. Namekawa, Y. Taniguchi, N. Ukita, T. Yamazaki, and T. Yoshié (PACS Collaboration), Phys. Rev. D 101, 094504 (2020).

[18] S. Aoki et al. (Flavor Lattice Averaging Group), Eur. Phys. J. C 80, 113 (2020).

[19] W. J. Marciano, Phys. Rev. Lett. 93, 231803 (2004).

[20] F. Ambrosino et al. (KLOE Collaboration), Phys. Lett. B 632, 76 (2006).

[21] R. J. Dowdall, C. T. H. Davies, G. P. Lepage, and C. McNeile, Phys. Rev. D 88, 074504 (2013).

[22] N. Carrasco et al., Phys. Rev. D 91, 054507 (2015).

[23] A. Bazavov et al., Phys. Rev. D 98, 074512 (2018).
[24] N. Miller et al., Phys. Rev. D 102, 034507 (2020).

[25] N. Cabibbo, E. C. Swallow, and R. Winston, Phys. Rev. Lett. 92, 251803 (2004).

[26] Y.S. Amhis et al. (HFLAV Collaboration), Eur. Phys. J. C 81, 226 (2021).

[27] N. H. Christ, T. Izubuchi, C. T. Sachrajda, A. Soni, and J. Yu (RBC and UKQCD Collaborations), Phys. Rev. D 88, 014508 (2013).

[28] Z. Bai, N. H. Christ, T. Izubuchi, C. T. Sachrajda, A. Soni, and J. Yu, Phys. Rev. Lett. 113, 112003 (2014).

[29] N. H. Christ, X. Feng, G. Martinelli, and C. T. Sachrajda, Phys. Rev. D 91, 114510 (2015).

[30] N. H. Christ, X. Feng, A. Portelli, and C. T. Sachrajda (RBC and UKQCD Collaborations), Phys. Rev. D 92, 094512 (2015).

[31] N. H. Christ, X. Feng, A. Portelli, and C. T. Sachrajda (RBC and UKQCD Collaborations), Phys. Rev. D 93, 114517 (2016).

[32] N. H. Christ, X. Feng, A. Juttner, A. Lawson, A. Portelli, and C. T. Sachrajda, Phys. Rev. D 94, 114516 (2016).

[33] Z. Bai, N. H. Christ, X. Feng, A. Lawson, A. Portelli, and C. T. Sachrajda, Phys. Rev. D 98, 074509 (2018).

[34] N. H. Christ, X. Feng, A. Portelli, and C. T. Sachrajda (RBC and UKQCD Collaborations), Phys. Rev. D 100, 114506 (2019).

[35] N. H. Christ, X. Feng, L.-C. Jin, and C. T. Sachrajda, Phys. Rev. D 103, 014507 (2021).

[36] B. C. Tiburzi, M. L. Wagman, F. Winter, E. Chang, Z. Davoudi, W. Detmold, K. Orginos, M. J. Savage, and P. E. Shanahan, Phys. Rev. D 96, 054505 (2017).

[37] P. E. Shanahan, B. C. Tiburzi, M. L. Wagman, F. Winter, E. Chang, Z. Davoudi, W. Detmold, K. Orginos, and M. J. Savage, Phys. Rev. Lett. 119, 062003 (2017).

[38] A. Nicholson et al., Phys. Rev. Lett. 121, 172501 (2018).

[39] X. Feng, L.-C. Jin, X.-Y. Tuo, and S.-C. Xia, Phys. Rev. Lett. 122, 022001 (2019).

[40] X.-Y. Tuo, X. Feng, and L.-C. Jin, Phys. Rev. D 100, 094511 (2019).

[41] W. Detmold and D. J. Murphy (NPLQCD Collaboration), arXiv:2004.07404.

[42] X. Feng, L.-C. Jin, Z.-Y. Wang, and Z. Zhang, Phys. Rev. D 103, 034508 (2021).

[43] Z. Davoudi and S. V. Kadam, Phys. Rev. D 102, 114521 (2020). 
[44] Z. Davoudi and S. V. Kadam, Phys. Rev. Lett. 126, 152003 (2021).

[45] M. T. Hansen, H. B. Meyer, and D. Robaina, Phys. Rev. D 96, 094513 (2017).

[46] S. Hashimoto, Prog. Theor. Exp. Phys. 2017, 053 B03 (2017).

[47] P. Gambino and S. Hashimoto, Phys. Rev. Lett. 125, 032001 (2020).

[48] N. Carrasco, V. Lubicz, G. Martinelli, C. T. Sachrajda, N. Tantalo, C. Tarantino, and M. Testa, Phys. Rev. D 91, 074506 (2015).

[49] V. Lubicz, G. Martinelli, C. T. Sachrajda, F. Sanfilippo, S. Simula, and N. Tantalo, Phys. Rev. D 95, 034504 (2017).

[50] D. Giusti, V. Lubicz, G. Martinelli, C. T. Sachrajda, F. Sanfilippo, S. Simula, N. Tantalo, and C. Tarantino, Phys. Rev. Lett. 120, 072001 (2018).

[51] X. Feng and L. Jin, Phys. Rev. D 100, 094509 (2019).

[52] N. H. Christ, X. Feng, J. Lu-Chang, and C. T. Sachrajda, Proc. Sci., LATTICE2019 (2020) 259.

[53] A. Desiderio et al., Phys. Rev. D 103, 014502 (2021).

[54] R. Frezzotti, M. Garofalo, V. Lubicz, G. Martinelli, C. T. Sachrajda, F. Sanfilippo, S. Simula, and N. Tantalo, Phys. Rev. D 103, 053005 (2021).

[55] X.-Y. Tuo, X. Feng, L.-C. Jin, and T. Wang, arXiv:2103 .11331 .

[56] C.-Y. Seng, X. Feng, M. Gorchtein, L.-C. Jin, and U.-G. Meißner, J. High Energy Phys. 10 (2020) 179.

[57] V. Cirigliano, M. Knecht, H. Neufeld, H. Rupertsberger, and P. Talavera, Eur. Phys. J. C 23, 121 (2002).

[58] V. Cirigliano, M. Giannotti, and H. Neufeld, J. High Energy Phys. 11 (2008) 006.
[59] A. Sirlin, Rev. Mod. Phys. 50, 573 (1978); 50, 905(E) (1978).

[60] A. Sirlin, Phys. Rev. 164, 1767 (1967).

[61] D. H. Wilkinson and B. E. F. Macefield, Nucl. Phys. A158, 110 (1970).

[62] C.-Y. Seng, D. Galviz, and U.-G. Meißner, J. High Energy Phys. 02 (2020) 069.

[63] J. Erler, Rev. Mex. Fis. 50, 200 (2004).

[64] M. Di Carlo, D. Giusti, V. Lubicz, G. Martinelli, C. T. Sachrajda, F. Sanfilippo, S. Simula, and N. Tantalo, Phys. Rev. D 100, 034514 (2019).

[65] V. Cirigliano, M. Knecht, H. Neufeld, and H. Pichl, Eur. Phys. J. C 27, 255 (2003).

[66] S. Descotes-Genon and B. Moussallam, Eur. Phys. J. C 42, 403 (2005).

[67] S. A. Larin and J. A. M. Vermaseren, Phys. Lett. B 259, 345 (1991).

[68] P. A. Baikov, K. G. Chetyrkin, and J. H. Kuhn, Phys. Rev. Lett. 104, 132004 (2010).

[69] T. Blum et al. (RBC and UKQCD Collaborations), Phys. Rev. D 93, 074505 (2016).

[70] Y. Li, S.-C. Xia, X. Feng, L.-C. Jin, and C. Liu, Phys. Rev. D 103, 014514 (2021).

[71] W. Detmold, D. J. Murphy, A. V. Pochinsky, M. J. Savage, P. E. Shanahan, and M. L. Wagman, arXiv:1908.07050.

[72] X. Feng, Y. Fu, and L.-C. Jin, Phys. Rev. D 101, 051502 (2020).

[73] K. G. Chetyrkin, J. H. Kuhn, and M. Steinhauser, Comput. Phys. Commun. 133, 43 (2000).

[74] B. Ananthanarayan and B. Moussallam, J. High Energy Phys. 06 (2004) 047. 